\title{
BMJ Open Study protocol for a randomised double-blinded, sham-controlled, prospective, cross-over clinical trial of vagal neuromodulation for pain treatment in patients with chronic pancreatitis
}

Janusiya Anajan Muthulingam,, ${ }^{1,2}$ Søren Schou Olesen, ${ }^{2,3}$ Tine Maria Hansen, ${ }^{1,2}$ Christina Brock, ${ }^{2,4}$ Asbjørn Mohr Drewes, ${ }^{2,3}$ Jens Brøndum Frøkjær ${ }^{\bullet 1,2}$

To cite: Muthulingam JA, Olesen SS, Hansen TM, et al. Study protocol for a randomised double-blinded, sham-controlled, prospective, cross-over clinical trial of vagal neuromodulation for pain treatment in patients with chronic pancreatitis. BMJ Open 2019;9:e029546. doi:10.1136/ bmjopen-2019-029546

- Prepublication history for this paper is available online. To view these files please visit the journal online (http://dx.doi. org/10.1136/bmjopen-2019029546).

Received 31 January 2019 Revised 4 June 2019 Accepted 21 June 2019

Check for updates

(c) Author(s) (or their employer(s)) 2019. Re-use permitted under CC BY-NC. No commercial re-use. See rights and permissions. Published by BMJ.

For numbered affiliations see end of article.

Correspondence to

Professor Jens

Brøndum Frøkær;; jebf@rn.dk

\section{ABSTRACT}

Introduction The management of chronic pancreatitis (CP) is challenging and requires a personalised approach focused on the individual patient's main symptoms. Abdominal pain is the most prominent symptom in $\mathrm{CP}$, where central pain mechanisms, including sensitisation and impaired pain modulation, often are involved. Recent clinical studies suggest that vagal nerve stimulation (VNS) induces analgesic effects through the modulation of central pain pathways. This study aims to investigate the effect of 2 weeks transcutaneous VNS (t-VNS) on clinical pain in patients with $\mathrm{CP}$, in comparison to the effect of sham treatment.

Methods and analysis Twenty-one patients with CP will be enrolled in this randomised, double-blinded, singlecentre, sham-controlled, cross-over study. The study has two treatment periods: A 2-week active t-VNS using GammaCore device and a 2-week treatment with a sham device. During both treatment periods, the patients are instructed to self-administer VNS bilaterally to the cervical vagal area, three times per day. Treatment periods will be separated by 2 weeks. During the study period, patients will record their daily pain experience in a diary (primary clinical endpoint). In addition, all subjects will undergo testing which will include MRI, quantitative sensory testing, cardiac vagal tone assessment and collecting blood samples, before and after the two treatments to investigate mechanisms underlying VNS effects. The data will be analysed using the principle of intention to treat. Ethics and dissemination The regional ethics committee has approved the study: $\mathrm{N}-20170023$. Results of the trial will be submitted for publication in peer-reviewed journals. Trial registration number The study is registered at www.clinicaltrials.gov: NCT03357029.

\section{INTRODUCTION}

Chronic pancreatitis (CP) is a disease characterised by progressive pancreatic inflammation and fibrosis, resulting in damage to and loss of exocrine (acinar), endocrine (islet

\section{Strengths and limitations of the study}

- This is the first study to examine the analgesic effect of transcutaneous vagal nerve stimulation (t-VNS) in patients with chronic pancreatitis with abdominal pain.

- A randomised double-blinded, sham-controlled, prospective cross-over design will be used with both clinical and experimental outcomes, which allow for the exploration of the mechanisms underlying putative clinical effects.

- The study investigates the effect of 2 weeks of $t-V N S$ treatment; hence, further studies are needed to explore long-term effects.

- The single-centre design may limit generalisability of the study results.

cells) and ductal cells. ${ }^{1}$ Chronic abdominal pain is the dominating symptom in $\mathrm{CP}$ and is present in up to $70 \%$ of patients. ${ }^{2}$ Pain is associated with reduced quality of life, increased hospitalisation frequencies and thus a significant socioeconomic burden. ${ }^{3}$

The aetiology of pain in CP is increasingly better understood and often involves multiple mechanisms in the individual patient. In addition to local pathology in the pancreatic gland and its surrounding tissues, central pain pathways undergo neuroplastic changes during the course of CP. These involve sensitisation of central pain pathways, functional and structural reorganisation of the brain as well as impaired efficacy of endogenous pain modulatory pathways. These neural abnormalities can be targeted by different pharmacological therapies, but their effect is often limited and associated with significant side-effects in many patients. 


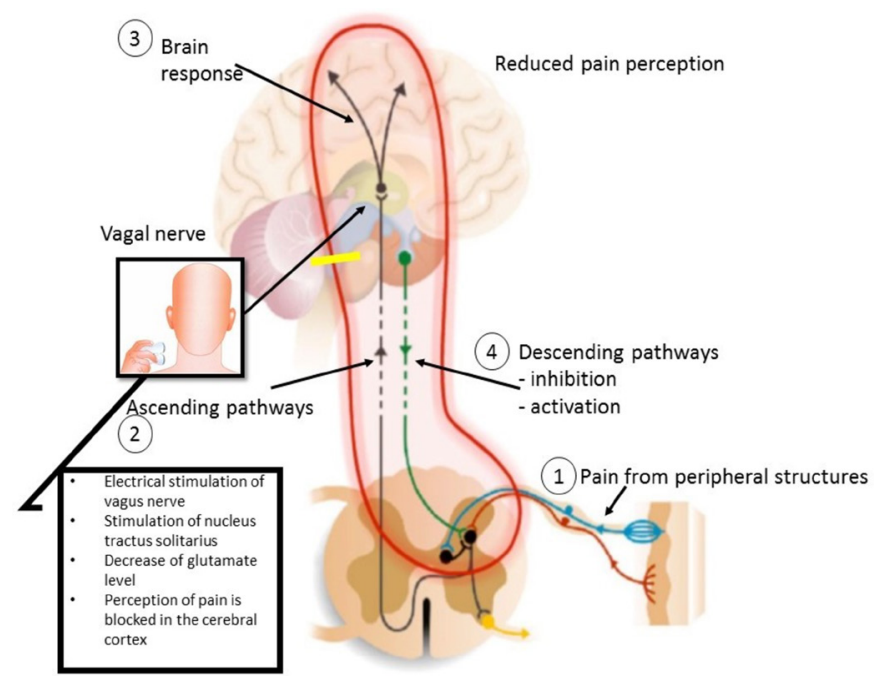

Figure 1 Mode of action of transcutaneous vagal nerve stimulation (t-VNS). (1) Pain arises in the periphery, for example, pancreas and a signal is sent to the spinal cord. This leads to the ascending activation of the spinal neurons (2). In the brain, pain is processed in higher cortical centres (3). t-VNS, it is expected to block the perception of pain in the cerebral cortex, by stimulating nucleus tractus solitarius and thereby decrease glutamate level. Simultaneously, the net-descending inhibition will be activated as a result of topdown input from cortex and the limbic system (4).

This has led to an increased interest in complementary treatment modalities for pain in patients with CP. In a model of oesophageal hyperalgesia, we have shown that physiological deep breathing enhanced vagal tone, which in response increased the pain detection threshold. ${ }^{4}$ In addition, this effect was abolished by atropine administration thereby proving that enhanced parasympathetic tone leads to the prevention of oesophageal pain hypersensitivity. ${ }^{4}$ Also, we have previously shown an improved gastrointestinal motility and decreased pain sensitivity following non-invasive vagal nerve stimulation (VNS) of the auricular branch of the vagal nerve in conjunction with a deep-breathing approach in healthy subjects. ${ }^{5}$ Another non-pharmacological treatment modality is transcutaneous vagal nerve stimulation (t-VNS), in which short bursts of electrical energy are directed onto the vagal nerve at the neck ${ }^{6}$ (figure 1). $\mathrm{t}-\mathrm{VNS}$ has been shown to induce analgesic ${ }^{57}$ and anti-inflammatory effects in healthy individuals ${ }^{8}$ and different diseases. The exact mechanisms by which VNS modulates chronic pain are unclear; however, it has been proposed that the analgesic effect is potentially mediated by vagal afferents that inhibit spinal nociceptive reflexes and transmission. ${ }^{9}$ Specifically, the analgesic effects are mediated through vagal afferent modulation in the nucleus tractus solitaries, raphe magnus, locus ceruleus, amygdala and periaqueductal grey, which are involved in the descending inhibition of pain. ${ }^{9}{ }^{10}$ It has also been demonstrated that VNS inhibits spinal cord neurons below C3 but excites neurons between C1 and C3, suggesting that propriospinal neurons from high segments play an essential role in vagally mediated antinociception. Thus, VNS appears to induce neuromodulatory antinociception through peripheral and central, ascending and descending pathways. ${ }^{11}$ Also, the non-pharmacological treatment is FDA approved for the acute treatment in patients with migraine. $^{12}$

This study aims to examine the analgesic effect of a 2-week t-VNS in patients with CP and to explore the underlying analgesic mechanisms using advanced neuroimaging techniques and quantitative sensory testing (QST). We hypothesised that 2-week t-VNS treatment will induce clinically relevant pain relief compared with sham treatment, and that these effects are mediated via the modulation of central pain pathways. To answer the overall study aims, we have two clinical and two experimental objectives:

1. The primary clinical objective is to assess the effect of $\mathrm{t}-\mathrm{VNS}$ on the daily pain experience documented in a pain diary in patients with CP.

2. Secondary clinical objectives are to document changes in quality of life and daily functioning.

3. The experimental objective is to assess the effect of $\mathrm{t}$-VNS on (A) resting state brain function assessed by MRI, and (B) brain metabolites assessed by magnetic resonance spectroscopy.

4. Secondary experimental objectives are to assess the effect of t-VNS on (A) experimental pain stimuli documented by QST, (B) cardiac vagal tone (CVT) and (C) proinflammatory cytokine profiles obtained from blood samples.

\section{METHODS AND ANALYSES \\ Study design}

Randomised, single-centre, double-blinded, prospective, sham-controlled, cross-over study. The trial will be performed at Aalborg University Hospital and will be reported according to the Consolidated Standards of Reporting Trials. ${ }^{13}$ The study protocol follows the Standard Protocol Items: Recommendations for Interventional Trials statement. ${ }^{14}$

All patients undertake the t-VNS treatment using an active GammaCore-S, 300 treatments (10009-00603) device (ElectroCore LLC, Basking Ridge, New Jersey, USA) and sham treatment using a sham device (10009$00603 \mathrm{P}$ ) which is identical in appearance to GammaCore.

Half of the patients will be randomised to start with 2-week t-VNS treatment, followed by a 2-week washout period. Then, this group will be reallocated to sham treatment. The other half of patients will do the study periods in opposite order (sham treatment followed by $\mathrm{t}$-VNS treatment). The 2-week washout period has been used in the previous studies of transcranial neuromodulation $^{15}$ and was shown to be sufficient to reset the effects of neuromodulation. ${ }^{16}$ Each patient will be scheduled for four identical hospital visits (before and after each treatment period). The visits consist of (1) fulfilment of questionnaires, (2) collection of blood samples, (3) brain 
Timeline

Inclusion
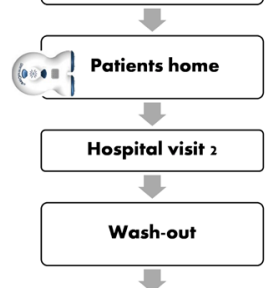

Hospital visit 3

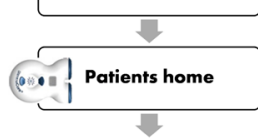

Hospital visit 4
Study design

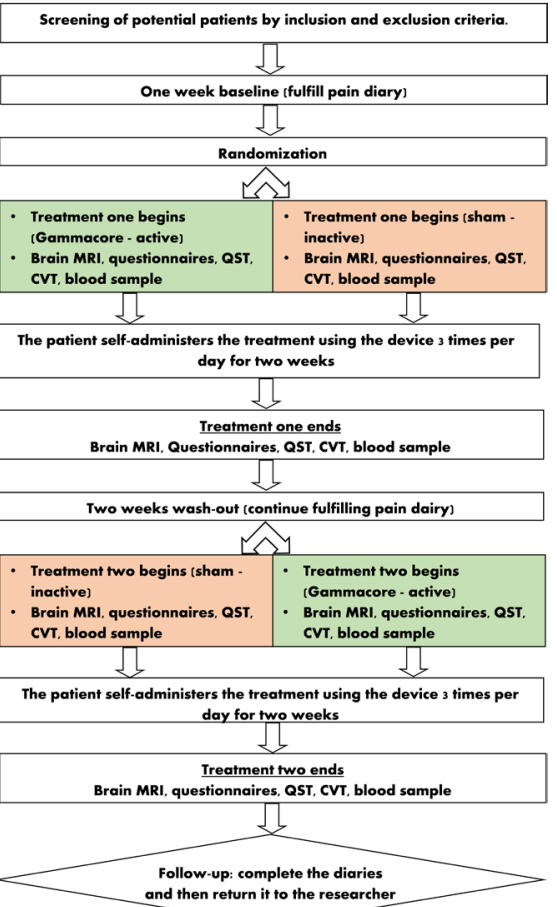

Figure 2 Schematic flow chart of the interventional study design enabling comparison of the modulatory effect to self-administered of transcutaneous vagal nerve stimulation (t-VNS) in patients with chronic pancreatitis. Patients with chronic pancreatitis will be randomly assigned to one of two double-blinded treatments: (1) 2 weeks of t-VNS, 2 weeks of washout and 2 weeks of sham treatment; or (2) 2 weeks of sham treatment, 2 weeks of washout and 2 weeks of $\mathrm{t}-\mathrm{VNS}$. Evaluation of the two treatments will be assessed by collecting pain diary, pain questionnaires, MRI scan, blood sample, cardiac vagal tone (CVT) and pain assessments. quantitative sensory testing (QST).

MRI scan, (4) QST and (5) assessment of CVT (figure 2, table 1).

\section{Study participants}

Patients will be recruited via personal correspondence and during visits at the outpatient clinic. Patients who agree to participate in the study and fill in an informed consent will be invited to participate in the study. A screening session and physical examination prior to inclusion will be conducted by a medical doctor including relevant medical and medication history and screening against the eligibility criteria. All patients will be asked to continue their medication during the entire study, and any changes needed in pain medication will be noted in the diary.

\section{Inclusion criteria and exclusion criteria}

Patients from the age of 18 years will be included in the study. They will have a clinical diagnosis of CP based on the Mayo clinical diagnostic criteria. ${ }^{17}$ All aetiological types of patients with $\mathrm{CP}$ would be included (including alcohol, nicotine, hereditary, efferent duct factors and immunological aetiologies). The patients must suffer from chronic abdominal pain characteristics for CP, meet the criteria for chronic pain (pain $\geq 3$ days per week for at least 3 months) and must consider their pain as insufficiently treated with their prescribed analgesic treatment. Additionally, the patients must be willing and able to comply with the scheduled visits, treatment plan, laboratory tests and other study procedures. Finally, the patient must sign the informed consent and power of attorney document.

Patients will be excluded if they have any clinically significant abnormalities that may increase the risk associated with trial participation or may interfere with the interpretation of the trial results. Also, patients with alcohol and illegal drug dependence, cardiovascular diseases, low blood pressure $(<100 / 60 \mathrm{~mm} \mathrm{Hg})$, elevated intracranial pressure will be excluded. Additionally, patients who are participating in another intervention study, patients who are pregnant or intend to become pregnant and patients who suffer from painful conditions other than CP that make them unable to distinguish the pain associated with CP from chronic pain of other origin will be excluded. Patients will also be excluded if there are any contraindications for MRI (including cardiac pacemaker, implantable metallic components, etc.), have known neuropathy or previous vagal nerve surgery (table 1 ).

Participants can withdraw from the study at any time they may wish. Patients will be withdrawn from the study if they do not meet for the scheduled study visits or miss a treatment period, and if they do not maintain inclusion/ exclusion criteria.

\section{Interventions}

Study interventions are t-VNS treatment and sham treatment (figures 2 and 3). Prior to receiving the t-VNS treatment/sham treatment, the standard care must be stable.

Patients will be thoroughly instructed to use the device, and when the healthcare providers are confident that the patient is capable of using the device independently, the device will be handed over to the patient. t-VNS is administered by using a handheld device (GammaCore; ElectroCore LLC), which consists of a battery-powered portable stimulator with a digitally controlled user interface that controls the signal amplitude and two gel-covered (Sigma gel, Parker Laboratories, New Jersey, USA) contact electrodes which deliver electrical stimulation to the cervical vagal nerve. One dose corresponds to $120 \mathrm{~s}$ of t-VNS to the left cervical vagal nerve followed by $120 \mathrm{~s}$ of t-VNS to the right cervical vagal nerve, with the amplitude of simulation titrated to achieve mild pulling of the ipsilateral oral commissure. ${ }^{18}$ Bilateral stimulation has shown to be effective in previous studies with GammaCore. ${ }^{19} 20$ The patient self-administers the treatment, using the device at home three times per day (morning, afternoon and evening) for 2 weeks. Previous studies with Gamma-Core have shown that three doses per day have been effective. ${ }^{121}$ 
Table 1 Trial characteristics based on WHO trial registration dataset

\begin{tabular}{ll}
\hline Data category & Trial information \\
\hline Primary registry and trial & ClinicalTrials.gov (NCT03357029) \\
identifying number
\end{tabular}

identifying number

Date of registration in 29 November 2017

primary registry

Secondary identifying North Denmark Region Committee on Health Research Ethics: protocol number N-20170023

numbers

Source(s) of monetary or The study is conducted as a sponsor-investigator initiated study with financial support from material support Independent Research Fund Denmark (DFF: 7016-00073).

\begin{tabular}{ll} 
Primary sponsor & JBF \\
\hline Secondary sponsor & NA \\
\hline Contact for public queries & JBF \\
$\begin{array}{ll}\text { Contact for scientific } \\
\text { queries }\end{array}$ & JBF \\
\hline
\end{tabular}

$\begin{array}{ll}\text { Public title } & \text { Neuromodulation in patients with painful chronic pancreatitis (CP) } \\ \text { Scientific title } & \begin{array}{l}\text { Study protocol for a randomised double-blinded, sham-controlled, prospective, cross-over clinical } \\ \text { trial of vagal neuromodulation for pain treatment in patients with CP }\end{array}\end{array}$

$\begin{array}{ll}\text { Country of recruitment } & \text { Denmark } \\ \text { Healthy conditions(s) or } & \text { CP } \\ \text { problems studied } & \end{array}$

\section{Interventions 2-week transcutaneous vagal nerve stimulation (t-VNS) on the cervical vagal area (self-} administering vagal nerve stimulation bilaterally to the cervical vagal area, the times per day).

Key inclusion and exclusion criteria

Inclusion criteria: age $\geq 18$ years; patients with a diagnosis of $\mathrm{CP}$ diagnosed using the Mayo Clinic diagnostic criteria; the participants must be able to read and understand Danish; the patients must suffer from chronic abdominal pain characteristic for $\mathrm{CP}$, meet the criteria for chronic pain (pain $\geq 3$ days per week in at least 3 months) and must consider their pain as insufficiently treated with their usual analgesic treatment; personally, signed and dated informed consent document and the power of attorney document; patients willing and able to comply with the scheduled visits, treatment plan, laboratory tests and other trial procedures. Exclusion criteria: patients with any clinically significant abnormalities that in the opinion of the investigator may increase the risk associated with trial participation or may interfere with the interpretation of the trial results; alcohol dependence; illegal drug dependencies; participating in another study where investigational drug is used; patients must not suffer from painful conditions other than CP that make them unable to distinguish the pain associated with $\mathrm{CP}$ from the chronic pain of other origin; cardiovascular diseases; low blood pressure<100/60, not able to understand or follow the instructions; any condition with elevated intracranial pressure; female patients who are pregnant; contraindications for MRI; previous surgery on vagal nerve; known neuropathy.

\begin{tabular}{ll} 
Study type & $\begin{array}{l}\text { Interventional allocation: randomised } \\
\text { Masking: double blind } \\
\text { Assignment: crossover } \\
\text { Primary purpose: treatment }\end{array}$ \\
\hline Date of first enrolment & January 2018 \\
\hline Target sample size & 21 \\
\hline Recruitment status & Recruiting \\
\hline Primary outcome(s) & Change in NRS scores in pain diary \\
\hline Key secondary outcomes & $\begin{array}{l}\text { Assessment of the effect of t-VNS on (A) resting state brain function assessed by MRI, and (B) } \\
\text { (s) }\end{array}$ \\
\hline
\end{tabular}

DFF, Danmarks Frie Forskningsfond (Independent Research Fund Denmark); MR, magnetic resonance; NRS, Numeric Rating Scale.

The stimulation device is positioned anterior to the sternocleidomastoid muscle, over the carotid artery as this runs in close proximity with the vagal nerve. The active GammaCore device produces a low-voltage electrical signal comprising a $5 \mathrm{kHz}$ sine wave burst lasting for $1 \mathrm{~ms}$ (five sine waves, each lasting $0.2 \mathrm{~ms}$ ), with such bursts repeated once every $40 \mathrm{~ms}(25 \mathrm{~Hz})$, generating a $24 \mathrm{~V}$ peak voltage and $60 \mathrm{~mA}$ peak output current. Those 


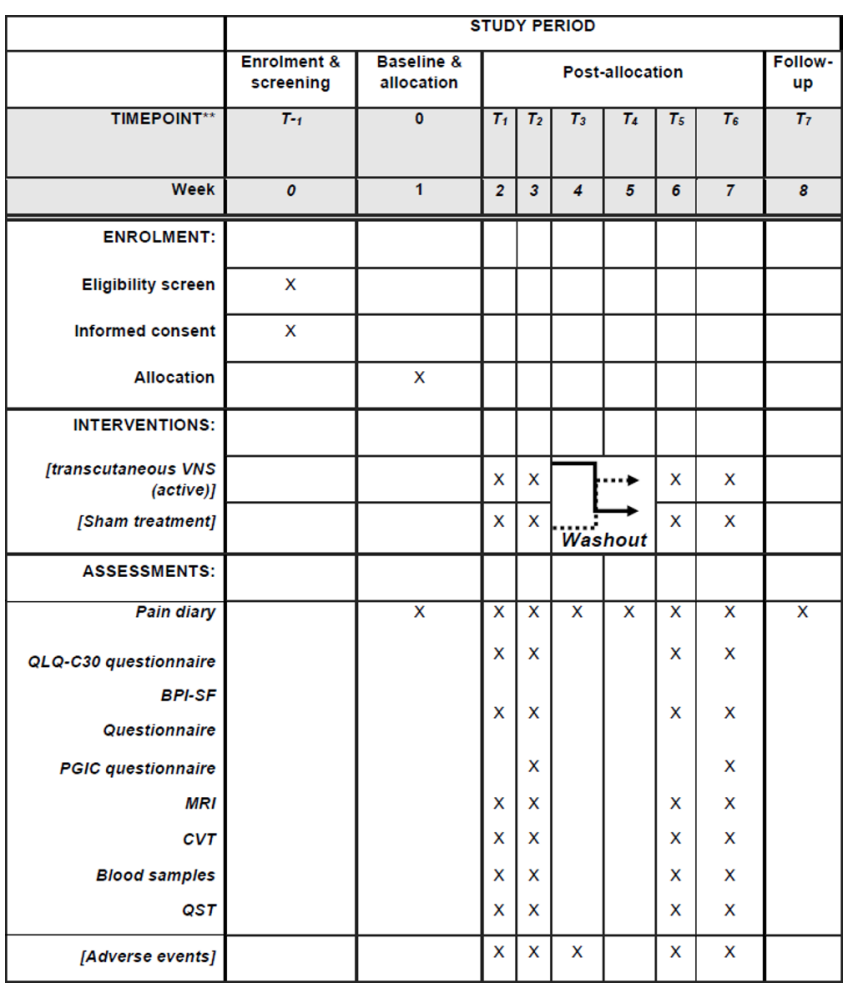

Figure 3 Standard Protocol Items: Recommendations for Interventional Trials figure. BPI-SF, Brief Pain InventoryShort Form; CVT, cardiac vagal tone; PGIC, Patient Global Impression of Changes Questionnaire; QST, quantitative sensory testing; VNS, vagal nerve stimulation.

parameters have been used to activate the vagal nerve in electrophysiological studies. ${ }^{1920}$

To mimic the sensation of the active treatment, the sham device will provide vibration. ${ }^{22}$ The appearance, weight, visual and audible feedback, and user application are identical for the sham and t-VNS devices. However, the sham device produces a low-frequency $(0.1 \mathrm{~Hz})$ biphasic signal that does not stimulate the vagal nerve or generally cause muscle contractions. ${ }^{23}$ Additionally, both devices will display a numeric value between 1 and 40, signifying the intensity of the stimulation. The maximum intensity per stimulation is 40 for both devices (figure 1). The intensity of the stimulation can vary from patient to patient. The intensity for stimulation is reached by increasing the stimulation to the maximum which the patient can tolerate without excessive pain. Some patients can tolerate less than other patients depending on the pain level. Therefore, the dosage of every stimulation is patient dependent. $^{24}$

Compliance will be assessed by reading the remaining doses displayed at the device after each treatment period. Additionally, the patients will be asked to keep a record of the stimulation intensity of the doses applied at each stimulation. In addition, questions on compliance will be asked after each treatment period. Finally, adherence will be recorded by patients' diary.

During the study periods, the patient will continue their standard care, without changes in their current pain treatment.
Randomisation, sequence generation and allocation concealment

Once eligibility and consent have been approved and completed, randomisation will occur using a randomisation list generated by an automatic web-based randomisation programme. Patients will be randomly assigned to VNS/sham or sham/VNS using block randomisation, allowing seven patients at the time to be randomised in equal proportions for the order of active t-VNS or sham stimulation. The randomisation order will be kept in closed envelopes; therefore, patients will get their assignment according to the order of entrance in the study. This process will be carried out by a member of the research team who is not involved in the recruitment process or conduction of the study.

An unblinded researcher will be involved in delivering the medical device according to the randomisation schedule. The sequence will follow a 1:1 sequential design, in a double-blinded fashion. Additionally, the outcome assessor (data analyst) will be blinded during the statistical analyses of experimental outcomes. A series of numbered, sealed, envelopes will be used to ensure concealed allocation.

\section{Blinding}

Both, active and sham devices are labelled with a serial number and not outwardly identified as active or sham. All researchers involved in the data collection and MRI analysis will be blinded to the treatment allocation group until after analyses are performed at the completion of the trial. Additionally, all patients are blinded, and they do not know that the sham treatment is an inactive treatment. Particularly, patients will be informed that they have to undergo two different interventions with two different devices, and the purpose of this study is to investigate the most effective treatment.

Manufacturing and preparation of the medical devices are handled by an external good manufacturing practice-accredited facility (ElectroCore). As the patients do not know that the sham treatment is an inactive treatment, we will not be able to ask the patient 'do you think you received active or inactive treatment?'; thus, we will not be able assess and determine if the blinding was effective.

Unblinding is only permissible if a patient experiences any serious adverse events and that the investigator/ doctor judge that it is essential to know the treatment allocation in order to treat the patient appropriately.

\section{Primary clinical outcome measures}

The primary clinical efficacy parameter to be evaluated is $30 \%$ pain relief. This is assessed as changes in the daily experience of pain, which will be measured using a patient pain diary based on the Numeric Rating Scale (NRS) (1=no pain, 10=worst pain imaginable). Patients will be asked to score daily pain levels in the diary for 8 weeks (including 1 week before the first treatment period and 1 week after the last study period, figures 2 and 3), with one NRS value for the average pain over the 
previous 24 hours and one NRS value for the worst pain over the previous 24 hours.

\section{Secondary clinical outcome measures}

Quality of Life Questionnaire, C30, V.3.0 (QoLQ-C30), ${ }^{25}$ the Brief Pain Inventory-Short Form (BPI-SF) Questionnaire $^{26}$ and Patient Global Impression of Changes ${ }^{27}$ Questionnaire (PGIC) are secondary clinical outcomes. Patient will complete QoLQ-C30 and BPI-SF Questionnaire before and after each treatment period, while the PGIC Questionnaire will only be fulfilled after the treatment periods. The QoLQ-C30 is composed of both multiitem scales and single-item measures. These include five functional scales, three symptom scales, a global health status and six single items. The BPI-SF Questionnaire rapidly assesses the severity of pain and its impact on daily functioning. Finally, the PGIC Questionnaire evaluates all aspects of patients' health and assess if there has been an improvement or decline in the overall clinical status.

\section{Experimental outcome measures}

Resting state functional MRI will be employed to detect brain activity and functional connectivity changes based on blood oxygen level-dependent (BOLD) signals before and after treatment of each patient. Additionally, magnetic resonance spectroscopy in anterior cingulate cortex, prefrontal cortex, parietal and insula will also be performed in order to investigate changes in brain metabolites before and after each treatment.

MRI data will be acquired on a 3 T MR scanner (Signa HDxt, General Electric, Milwaukee, Wisconsin, USA) equipped with an eight channel standard head coil. Scan time for a structural scan will be $5^{1 / 2} \mathrm{~min}$. Following parameters will be used for the structural scan: 150 slices, field of view (FOV) $250 \mathrm{~mm}$, echo time $3.6 \mathrm{~ms}$, repetition time $9.0 \mathrm{~ms}$, flip angle $14^{\circ}$, resolution $0.78 \times 0.78 \mathrm{~mm}$, matrix size $320 \times 320 \mathrm{~mm}$, slice thickness $1 \mathrm{~mm}$, full head coverage, with no gap. Functional scans will be acquired with following parameters: gradient echo, echo planar imaging, 192 volumes, $37-40$ slices, $\mathrm{FOV}=240 \mathrm{~mm}$, echo time $=30 \mathrm{~ms}$, repletion time $=2000 \mathrm{~ms}$, flip angle $=90^{\circ}$, matrix size $=64 \times 64$, resolution $=3.75 \times 3.75 \mathrm{~mm}$, slice thickness $3.8 \mathrm{~mm}$, no gap, axial slices. The scan time for functional MRI will be $6 \mathrm{~min}$ and $32 \mathrm{~s}$. Additionally, MRI spectroscopy will be used to estimate brain metabolites in the anterior cingulate cortex, prefrontal cortex, parietal and insula. For MRI spectroscopy, single voxel point resolved spectroscopy will be acquired. Following parameters will be used: echo time $=30 \mathrm{~ms}$, repetition time $=2000 \mathrm{~ms}$, scan time will be $5 \mathrm{~min}$ and the total number of scans will be 128 . Bandwidth will be $5000 \mathrm{~Hz}$. A $20 \times 20 \times 20 \mathrm{~mm}$ voxel of interest will be positioned on a sagittal T2-weighted fast spin echo sequence. Repletion time $=4600 \mathrm{~ms}$ and echo time $=102 \mathrm{~ms}$, matrix $384 \times 256$, slice thickness $3 \mathrm{~mm}$, gap $0.3 \mathrm{~mm}$, in the midline in the anterior cingulate cortex (ACC) with the inferior border along the anterior-posterior commissure line.
Secondary experimental outcome measures

Secondary outcomes are changes in QST, CVT and proinflammatory cytokine profiles.

QST includes temporal summation, ${ }^{28}$ pressure pain thresholds ${ }^{28} 29$ and conditioned pain modulation (CPM).$^{30}$ Temporal summation demonstrates an increase perception of pain to repetitive pain stimuli. ${ }^{28}$ Temporal summation will be recorded in the dermatome T10 (pancreatic area) and control area (dominant forearm) using the PinPrick stimulator, $256 \mathrm{mN}$ (MRC Systems GmbH Medizintechnische Systeme, Germany).

The pressure pain threshold and pressure pain tolerance will be determined by pressing an electronic pressure algometer (Somedic AB, Stockholm, Sweden) on specified muscle groups: C5—clavicula, T10—dorsum, T10-abdomen, L1-anterior superior iliac spine, and rectus femoris. Also, pressure pain threshold and pressure pain tolerance will be measured on bone. For the muscle pressure stimulation, the probe has a surface area of $1 \mathrm{~cm}^{2}$. Pressure will be increased at a rate of $30 \mathrm{kPa} / \mathrm{s}$ until the pressure pain threshold is reached. For the bone pressure stimulation, a probe with $3.1 \mathrm{~mm}^{2}$ (Aalborg University, Denmark) will be applied.

CPM is a clinically measurable form of descending pain modulation $^{30}$ that can be induced experimentally by a conditioning stimulus (the cold pressor test) and quantified by applying a 'test pain' (pressure stimulation of the right quadriceps musculature) before and after its induction. ${ }^{31}$ The patient will lower their dominant hand in cold water $\left(2^{\circ} \mathrm{C}\right.$ for maximum $\left.2 \mathrm{~min}\right)$. The difference in pressure stimulus intensity (pain threshold) before and after induction of cold pressor pain provides a quantitative index of CPM capacity for the individual patient. The techniques used for pressure stimulation and cold pressor test described above will be combined to measure CPM.

CVT is a beat-to-beat measure of brainstem efferent vagal activity, which is assessed by heart rate variability measurement and reflects the contribution of the vagal nerve to cardiac functioning. In this particular test, changes in $\mathrm{R}-\mathrm{R}$ interval would be measured non-invasively using eMotion Faros 180 device. ${ }^{32}$

Blood samples are collected to explore changes in proinflammatory cytokines profiles. $26 \mathrm{~mL}$ blood is collected, and the following inflammatory state and macrophage markers will be assessed: interferon-G, interleukin 8 (IL-8), IL-10, IL-12p70, IL-13, IL-1b, IL-2, IL-4, IL-6, tumour necrosis factor- $\alpha$ (TNF- $\alpha)$, monocyte chemoattractant protein- 1 and high sensitivity $\mathrm{C}$ reactive protein.

\section{Statistical power}

The study is powered to detect a minimal difference between the sham treatment and the active treatment of $30 \%$ on the average clinical pain score at the end of the two study periods. Based on a SD of $40 \%$, we determine that a study with 16 patients in a cross-over design is needed, with a power of $80 \%$, and the use of a two-sided 
significance level of 0.05 (alpha). This calculation (SD) is based on data from a study with patients with CP, who received pregabalin treatment, which related to an improvement in clinical measures of the pain score. ${ }^{33}$ To allow for a dropout rate of $25 \%$, we will aim to recruit 21 patients with CP. The sample size was calculated using statistical software package STATA V.15.0 (StataCorp LP, College Station). ${ }^{34}$

\section{Harms and adverse events}

We do not anticipate this project causing any harm or discomfort to the patients, and we will ensure that our patients participate in the study voluntarily.

Information about adverse events and serious adverse events will be collected from the date of inclusion and in all following contacts with the study subject throughout the project. Adverse events will be documented on the patient file and on the electronic case report form. All types of adverse events will be notified to the device manufacturer ElectroCore and to the Danish Health Authorities by use of Manufactures Incident Report Form.

\section{Data collection and data management}

All instruments in the questionnaires are validated. ${ }^{25} 26$ Additionally, all data collectors are highly experienced registered research nurses, radiographers and researchers who have been trained in good clinical practice (GCP). There will be regular meetings between the data collectors, monitor, principal investigator and other coresearchers involved in the project. All paper protocols will be kept safe and transferred to a computerised database. The questionnaires will be checked for errors and missing data by research staff. Data entries are double checked against the paper questionnaires.

During trial conduct, the GCP unit (GCP, Aalborg, Denmark) will conduct periodic monitoring of all signed consents at monitoring visits to ensure that the protocol and GCP standards are followed. The monitors may review source documents and medical records to confirm that data recorded on case report form is accurate. Thus, GCP monitoring includes all signed consents, signed power of attorney and adverse event (AE).

Criteria for the termination of the trial are when patients according to the sample size with valid data are recorded. If the study fails to recruit adequate patients according to the sample size by the end of 2019, the study will be terminated.

\section{Data analysis}

Both descriptive and analytical statistics will be used in order to compare groups and for analyses of outcomes over time including changes therein. All data will be presented as mean $\pm \mathrm{SD}$ and summarised in frequency tables, unless otherwise indicated. We will use Research Electronic Data Capture (REDCap) ${ }^{35}$ to store the data and the statistical software package STATA to perform statistical analysis. We will use mixed analysis of variance (ANOVA) for the inferential statistic of the parametric data, with Tukey's and/or Bonferroni post hoc tests for the primary clinical endpoints. Significance level will be set as $\alpha \leq 0.05$.

The principal analysis of clinical endpoints will be by intention to treat, meaning that all randomised patients are included in their initially assigned study arm, regardless of adherence to study protocol. Experimental endpoints will be analysed by per protocol, meaning that only patients completing the experimental set-up are included. The primary endpoints will be compared between the treatment groups.

Analysis of MRI data: we will use standard preprocessing procedures in Statistical Parametric Mapping (http:// www.fil.ion.ucl.ac.uk/spm/) before conducting the statistical analysis. Moreover, we will use a mixed effects design in which within-subject effects between the two treatments (before and after both treatments) responses brain activity and group effects will be modelled. For MR spectroscopy, specific metabolites changes will be assessed in pain-related brain regions. ${ }^{36}$

The rest of the data, like demographic data, change in circulating cytokines, and others, will be used descriptively and as input to regression and mixed-model analyses. The final statistical analysis plan, providing details of the analysis and presentation of the results, will be finalised before initiating any statistical analysis.

\section{Patient and public involvement}

The study was designed based on the need for new therapeutic options for patients with $\mathrm{CP}$ and the literature relating to pain management in $\mathrm{CP}$, as described in the introduction. The outcomes, such as pain scores and MRI brain scans, were deliberately chosen in order to assess the potential effect of t-VNS treatment both subjectively (patient oriented) and objectively. Furthermore, no patients were directly involved in the design, recruitment to or conduct of the study. However, an expert/chief doctor specialised in CP disease is an associated investigator of the study (SSO). The results and findings gathered from this study will be provided to the patients on request in the form of a written report.

There was no public involvement in the study design.

\section{DISCUSSION}

To the best of our knowledge, there are no randomised, sham-controlled, studies investigating the effect of t-VNS on clinical pain in patients with CP. We expect the study to provide clinical evidence of the analgesic effect of VNS and to elucidate its underlying mechanisms of action. This may pave the road for non-pharmacological treatment of pain associated with $\mathrm{CP}$ and the findings of the study may be generalisable to chronic pain conditions per se.

Previous studies have shown structural and functional alterations of the CNS in patients with $\mathrm{CP}$ with abdominal pain. ${ }^{37-41}$ The central nervous system (CNS) mechanisms may have the ability to recover by targeting treatment at 
plasticity mechanisms and reorganisation of neuronal pathways leading to the improvement of clinical symptoms. ${ }^{42}$ VNS treatment has emerged a promising technique in stimulating neural reorganisation and synaptic plasticity in cortical and subcortical networks, leading to the modulation of serotoninergic and noradrenergic pain inhibitory pathways. ${ }^{43}$ Those mechanisms might alter and regenerate the neural connectivity in regions responsible for pain. ${ }^{44-46}$ In addition, the vagal nerve serves as an essential transmitter of inflammatory signals in immune-to-neuronal communication. ${ }^{47-49}$ Afferent fibres of the vagal nerve relay information from viscera to the nucleus tractus solitaries in the brainstem, where it 'senses' proinflammatory cytokines such as IL-1, IL-6 and TNF- $\alpha$. Information is then projected to the parvocellular zone of the paraventricular nucleus of the hypothalamus, and therefore the comparison of functional alterations in the CNS and circulating levels of proinflammatory cytokines may provide evidence of an existing association. Some limitations about the study should be discussed. First, the patient group is very heterogeneous; they may suffer from comorbidities and may receive other pharmacological therapies, which may bias the results and consequently makes it difficult to assess the isolated effect of the VNS treatment. Second, the researchers may involuntarily become unblinded since the active treatment will deliver facial contractions while this is not present during sham treatment. Third, the relatively low number of patients may hamper the results including the explorative secondary outcomes; however, we eliminate the interindividual variability because of the cross-over design. Finally, although all the patients will be trained to use the device correctly according to manufactures' protocol, it is uncertain whether the patients will applicate the device correctly.

Regarding expected outcome, we hypothesise that VNS will reduce the pain in patients with $\mathrm{CP}$ and induce changes in pain-associated brain networks as well in the autonomic, inflammatory parameters and in the sensory system. Also, we expect that the neuromodulation will improve the overall quality of life in patients with CP.

\section{CONSENT TO PARTICIPATE}

The procedures set out in this study protocol, pertaining to conduct the study in compliance with GCP (CPMP/ ICH/135/95), designated Standard Operating Procedures, the Danish Health and Medicines Authority, the Research Ethics Committee in Denmark, and within the principles of the World Medical Association, Declaration of Helsinki amended by the 52nd General Assembly, Edinburgh, Scotland, October 2000, clarified by the General Assembly in Washington 2002, Tokyo 2004, and Seoul 2008 and Fortaleza 2013 as outlined herein.

Investigators (ie, medical doctors) will obtain informed consent from each patient. We will conduct this study under the rules of Resolution 466/12 and Declaration of Helsinki. Data will be stored electronically in REDCap database, with secure and restricted access. Data transfer will be encrypted and any information capable of identifying individuals removed. Results gathered from this protocol will be presented at national and international conferences and will be published in peer-reviewed journals. All confidential patient data will be protected, and patient identity will not be disclosed. Further dissemination of the dataset can be decided by the principal investigator.

Only researchers involved in the data collection and/or data analysis will have access to the final dataset. However, the principal investigator allows direct access to all source data and documents at monitoring, and inspection from the North Denmark Region Committee on Health Research Ethics, the Danish Health and Medicines Authority or from other countries' health authorities.

\section{Trial status}

The recruitment of the study started in January 2018. As of January 2019, a total of 13 patients have completed the study.

\section{Author affiliations}

${ }^{1}$ Mech-Sense, Department of Radiology, Aalborg University Hospital, Aalborg, Denmark

${ }^{2}$ Department of Clinical Medicine, Aalborg University, Aalborg, Denmark ${ }^{3}$ Mech-Sense, Department of Gastroenterology and Hepatology, Aalborg University Hospital, Aalborg, Denmark

${ }^{4}$ Aalborg University Hospital, Aalborg, UK

Contributors All authors conceived and designed the study and participated in the logistical planning of the study. JAM drafted the initial version of the manuscript and is collecting the data. All authors made significant contributions to the development and conceptualisation of the protocol. All authors reviewed the draft versions of the manuscript and have read and approved the final manuscript.

Funding The study is conducted as a sponsor-investigator initiated study with financial support from Independent Research Fund Denmark (DFF: 7016-00073).

Competing interests None declared.

Patient consent for publication Not required.

Provenance and peer review Not commissioned; externally peer reviewed.

Open access This is an open access article distributed in accordance with the Creative Commons Attribution Non Commercial (CC BY-NC 4.0) license, which permits others to distribute, remix, adapt, build upon this work non-commercially, and license their derivative works on different terms, provided the original work is properly cited, appropriate credit is given, any changes made indicated, and the use is non-commercial. See: http://creativecommons.org/licenses/by-nc/4.0/.

\section{REFERENCES}

1. Muniraj T, Aslanian HR, Farrell J, et al. Chronic pancreatitis, a comprehensive review and update. Part I: epidemiology, etiology, risk factors, genetics, pathophysiology, and clinical features. Dis Mon 2014;60:530-50.

2. Pham A, Forsmark C. Chronic pancreatitis: review and update of etiology, risk factors, and management. F1000Res 2018;7:607.

3. Mullady DK, Yadav D, Amann ST, et al. Type of pain, pain-associated complications, quality of life, disability and resource utilisation in chronic pancreatitis: a prospective cohort study. Gut 2011;60:77-84.

4. Botha $C$, Farmer AD, Nilsson M, et al. Preliminary report: modulation of parasympathetic nervous system tone influences oesophageal pain hypersensitivity. Gut 2015;64:611-7.

5. Frøkjaer JB, Bergmann S, Brock C, et al. Modulation of vagal tone enhances gastroduodenal motility and reduces somatic pain sensitivity. Neurogastroenterol Motil 2016;28:592-8. 
6. Johnson RL, Wilson CG. A review of vagus nerve stimulation as a therapeutic intervention. J Inflamm Res 2018;11:203-13.

7. Napadow V, Edwards RR, Cahalan CM, et al. Evoked pain analgesia in chronic pelvic pain patients using respiratory-gated auricular vagal afferent nerve stimulation. Pain Med 2012;13:777-89.

8. Brock C, Brock B, Aziz Q, et al. Transcutaneous cervical vagal nerve stimulation modulates cardiac vagal tone and tumor necrosis factoralpha. Neurogastroenterol Motil 2017;29:e12999-7.

9. Chakravarthy K, Chaudhry H, Williams K, et al. Review of the uses of vagal nerve stimulation in chronic pain management. Curr Pain Headache Rep 2015;19:54.

10. Frangos E, Ellrich J, Komisaruk BR. Non-invasive access to the vagus nerve central projections via electrical stimulation of the external ear: fMRI evidence in humans. Brain Stimul 2015;8:624-36.

11. Yuan H, Silberstein SD. Vagus Nerve and Vagus Nerve Stimulation, a Comprehensive Review: Part II. Headache 2016;56:259-66.

12. Gaul C, Diener HC, Silver N, et al. Non-invasive vagus nerve stimulation for PREVention and acute treatment of chronic cluster headache (PREVA): a randomised controlled study. Cephalalgia 2016;36:534-46

13. Moher D, Hopewell S, Schulz KF, et al. CONSORT 2010 explanation and elaboration: updated guidelines for reporting parallel group randomised trials. Int J Surg 2012;10:28-55.

14. Chan AW, Tetzlaff JM, Gøtzsche PC, et al. SPIRIT 2013 explanation and elaboration: guidance for protocols of clinical trials. BMJ 2013;346:e7586

15. Vitória A, De AL, Ribeiro V, et al. Effects of high-frequency transcranial magnetic stimulation on functional performance in individuals with incomplete spinal cord injury : study protocol for a randomized controlled trial. 2017:1-11.

16. Kuppuswamy A, Balasubramaniam AV, Maksimovic R, et al. Action of $5 \mathrm{~Hz}$ repetitive transcranial magnetic stimulation on sensory, motor and autonomic function in human spinal cord injury. Clin Neurophysiol 2011;122:2452-61.

17. Layer $P$, Yamamoto $H$, Kalthoff $L$, et al. The different courses of early- and late-onset idiopathic and alcoholic chronic pancreatitis. Gastroenterology 1994;107:1481-7.

18. Strickland I, Mwamburi M, Davis S, et al. Noninvasive vagus nerve stimulation in a primary care setting: effects on quality of life and utilization measures in multimorbidity patients with or without primary headache. Am J Manag Care 2018;24(24 Suppl):S517-S526.

19. Grazzi L, Tassorelli C, de Tommaso M, et al. Practical and clinical utility of non-invasive vagus nerve stimulation (nVNS) for the acute treatment of migraine: a post hoc analysis of the randomized, shamcontrolled, double-blind PRESTO trial. J Headache Pain 2018;19:1-9.

20. Tarn J, Legg S, Mitchell S, et al. The effects of noninvasive vagus nerve stimulation on fatigue and immune responses in patients with primary Sjögren's Syndrome. Neuromodulation 2018;2018.

21. Nesbitt AD, Marin JC, Tompkins E, et al. Initial use of a novel noninvasive vagus nerve stimulator for cluster headache treatment. Neurology 2015;84:1249-53.

22. De Icco R, Martinelli D, Bitetto V, et al. Peripheral vagal nerve stimulation modulates the nociceptive withdrawal reflex in healthy subjects: A randomized, cross-over, sham-controlled study. Cephalalgia 2018;38:1658-64.

23. Silberstein SD, Mechtler LL, Kudrow DB, et al. Non-Invasive Vagus Nerve Stimulation for the ACute Treatment of Cluster Headache: Findings From the Randomized, Double-Blind, Sham-Controlled ACT1 Study. Headache 2016;56:1317-32.

24. Use I, Risks P. Instructions for Use for gammaCore ${ }^{\circledR}-S$ 1: 1-28.

25. Fayers PM. EORTC QLQ-C30 scoring manual the EORTC QLQ-C30 introduction. EORTC QLQ-C30 Scoring Man EORTC QLQ-C30 Introd 2001;30:1-67.

26. Cleeland CS. Brief Pain Inventory (BPI). Cleel CS MD Anderson Cancer Cent 1982;1100:6.

27. Farrar JT, Young JP, LaMoreaux L, et al. Clinical importance of changes in chronic pain intensity measured on an 11-point numerical pain rating scale. Pain 2001;94:149-58.
28. Arendt-Nielsen L, Morlion B, Perrot S, et al. Assessment and manifestation of central sensitisation across different chronic pain conditions. Eur J Pain 2018;22:216-41.

29. Olesen SS, Bouwense SA, Wilder-Smith $\mathrm{OH}$, et al. Pregabalin reduces pain in patients with chronic pancreatitis in a randomized, controlled trial. Gastroenterology 2011;141:536-43.

30. Kennedy DL, Kemp HI, Ridout D, et al. Reliability of conditioned pain modulation: a systematic review. Pain 2016;157:2410-9.

31. Yarnitsky D, Bouhassira D, Drewes AM, et al. Recommendations on practice of conditioned pain modulation (CPM) testing. Eur $J$ Pain 2015;19:805-6.

32. Farmer AD, Coen SJ, Kano M, et al. Normal values and reproducibility of the real-time index of vagal tone in healthy humans: a multi-center study. Ann Gastroenterol 2014;27:362-8.

33. Trial C, Olesen SS, Bouwense SAW, et al. Clinical - pancreas pregabalin reduces pain in patients with chronic pancreatitis in a. YGAST 2011;141:536-43.

34. College Station TSL. StataCorp. 2015: Stata Stat Softw Release, 2013.

35. Harris PA, Taylor R, Thielke R, et al. Research electronic data capture (REDCap)--a metadata-driven methodology and workflow process for providing translational research informatics support. J Biomed Inform 2009;42:377-81.

36. Hansen TM, Olesen AE, Simonsen CW, et al. Cingulate metabolites during pain and morphine treatment as assessed by magnetic resonance spectroscopy. J Pain Res 2014;7:269-76.

37. Dimcevski G, Sami SA, Funch-Jensen P, et al. Pain in chronic pancreatitis: the role of reorganization in the central nervous system. Gastroenterology 2007;132:1546-56.

38. Frøkjær JB, Olesen SS, Gram M, et al. Altered brain microstructure assessed by diffusion tensor imaging in patients with chronic pancreatitis. Gut 2011;60:1554-62.

39. Frøkjær JB, Bouwense SA, Olesen SS, et al. Reduced cortical thickness of brain areas involved in pain processing in patients with chronic pancreatitis. Clin Gastroenterol Hepatol 2012;10:434-8.

40. Lelic D, Olesen SS, Graversen C, et al. Electrophysiology as a tool to unravel the origin of pancreatic pain. World $J$ Gastrointest Pathophysiol 2014;5:33-9.

41. Muthulingam J, Olesen SS, Hansen TM, et al. Progression of Structural Brain Changes in Patients With Chronic Pancreatitis and Its Association to Chronic Pain: A 7-Year Longitudinal Follow-up Study. Pancreas 2018;47:1267-76.

42. Apkarian AV, Hashmi JA, Baliki MN. Pain and the brain: specificity and plasticity of the brain in clinical chronic pain. Pain 2011;152(3 Suppl):S49-64

43. Garcia RG, Lin RL, Lee J, et al. Modulation of brainstem activity and connectivity by respiratory-gated auricular vagal afferent nerve stimulation in migraine patients. Pain 2017;158:1461-72.

44. O'Connell NE, Marston L, Spencer S, et al. Non-invasive brain stimulation techniques for chronic pain. Cochrane Database Syst Rev 2018;3:CD008208.

45. Seminowicz DA, Wideman TH, Naso L, et al. Effective treatment of chronic low back pain in humans reverses abnormal brain anatomy and function. $J$ Neurosci 2011;31:7540-50.

46. Čeko M, Shir Y, Ouellet JA, et al. Partial recovery of abnormal insula and dorsolateral prefrontal connectivity to cognitive networks in chronic low back pain after treatment. Hum Brain Mapp 2015;36:2075-92.

47. Bonaz B, Sinniger V, Pellissier S. Vagal tone: effects on sensitivity, motility, and inflammation. Neurogastroenterol Motil 2016;28:455-62.

48. Watkins LR, Maier SF, Goehler LE. Cytokine-to-brain communication: a review \& analysis of alternative mechanisms. Life Sci 1995;57:1011-26.

49. Hosoi T, Okuma Y, Matsuda T, et al. Novel pathway for LPS-induced afferent vagus nerve activation: possible role of nodose ganglion. Auton Neurosci 2005;120:104-7. 